\title{
Confirmation of a galaxy cluster hidden behind the Galactic bulge using the VVV survey
}

\author{
Georgina Coldwell ${ }^{1,2}$, Sol Alonso ${ }^{1,2}$, Fernanda Duplancic $^{1,2}$, Maren Hempel $^{3}$, \\ Valentin D. Ivanov ${ }^{4}$, and Dante Minniti ${ }^{3,5,6}$
}

\author{
${ }^{1}$ Consejo Nacional de Investigaciones Científicas y Técnicas (CONICET), Argentina \\ e-mail: georginacoldwell@gmail.com \\ 2 Departamento de Geofísica y Astronomía - Facultad de Ciencias Exactas, Físicas y Naturales - Universidad Nacional de San Juan, \\ San Juan, Argentina \\ 3 Instituto de Astrofísica, Pontificia Universidad Católica de Chile, Casilla 306, Santiago 22, Chile \\ ${ }^{4}$ European Southern Observatory, Alonso de Córdova 3107, Vitacura, Casilla 19001, Santiago, Chile \\ 5 Vatican Observatory, 00120 Vatican City State, Italy \\ ${ }^{6}$ Departamento de Ciencias Físicas, Universidad Andrés Bello, República 220, Santiago, Chile
}

Received 19 January 2014 / Accepted 30 June 2014

\section{ABSTRACT}

\begin{abstract}
Context. S uzaku and Chandra X-ray observations detected a new cluster of galaxies, Suzaku J1759-3450, at a redshift $z=0.13$. It is located behind the Milky Way, and the high Galactic dust extinction renders it nearly invisible at optical wavelengths.

Aims. We attempt here to confirm the galaxy cluster with near-infrared imaging observations and to characterize its central member galaxies.

Methods. Images from the VVV survey were used to detect candidate member galaxies of Suzaku J1759-3450 within the central region of the cluster up to $350 \mathrm{kpc}$ from the X-ray peak emission. Color-magnitude diagrams, color-color diagrams, and morphology criteria allowed us to select the galaxies among the numerous foreground sources.

Results. Fifteen candidate cluster members were found very close to a modeled red-sequence at the redshift of the cluster. Five members are extremely bright, and one is possibly a $\mathrm{cD}$ galaxy. The asymmetry in the spatial distribution of the galaxies with respect to the X-ray peak emission is an indicator that this cluster is still suffering a virialization process.

Conclusions. Our investigation of Suzaku J1759-3450 demonstrates the potential of the VVV survey to study the hidden population of galaxies in the zone of avoidance.
\end{abstract}

Key words. surveys - Galaxy: bulge - galaxies: clusters: individual: Suzaku J1759-3450

\section{Introduction}

Clusters of galaxies are the largest gravitationally bound systems in the Universe. Their number density and clustering strongly depend on cosmological parameters that provide potential means to constrain the underlying cosmological model (Bahcall et al. 2003; Gladders et al. 2007; Rozo et al. 2010). Clusters also harbor a large fraction of all galaxies and provide an outstanding environment that promotes their chemical evolution and morphological transitions. Consequently, the detection of new galaxy clusters enhances the possibility to unravel important clues about structure, galaxy formation, and evolution.

The thermal X-ray emission from the intracluster medium is a reliable tracer of galaxy clusters (Gursky et al. 1972). The detection of galaxy clusters using X-ray emission from hot gas is a popular technique (Romer et al. 2001; Pierre et al. 2006; Finoguenov et al. 2007). Mori et al. (2013) used this method to identify a rich galaxy cluster, Suzaku J1759-3450, behind the Milky Way bulge from Suzaku and Chandra observations. The spatial extent of the X-ray emission with a nearly circular shape, a radius of $\sim 4$ arcmin, and a bolometric X-ray luminosity of $L_{x}\left(r<r_{500}\right)=4.3 \times 10^{44} \mathrm{erg} \mathrm{s}^{-1}$ suggests that Suzaku J1759-3450 is a new cluster of galaxies at redshift $\mathrm{z}=0.13$. The peak of the surface brightness is located at $(\mathrm{RA}, \mathrm{Dec})_{\mathrm{J} 2000.0}=$ $\left(17^{\mathrm{h}} 59^{\mathrm{m}} 17^{\mathrm{s}} .41,34^{\circ} 50^{\prime} 18^{\prime \prime} \cdot 6\right)$ or $(l, b)=\left(356.3818^{\circ},-5.4660^{\circ}\right)$, and the total mass estimate was $2.2 \times 10^{14} M_{\odot}$ with an estimated core radius of $r_{\mathrm{c}}=1.61 \mathrm{arcmin}$. A precovery observation is available from the ROSAT Bright Source Catalogue (Voges et al. 1999).

Previous optical, near-infrared (NIR), X-ray, and H1 radio surveys have detected voids, galaxy clusters, and superclusters at low Galactic latitude (Woudt et al. 2004; Kraan-Korteweg \& Lahav 2000). Recently, a new NIR ESO public survey called VISTA Variables in Vía Láctea (VVV; Minniti et al. 2010; Saito et al. 2012) became available. Although the main scientific goals of VVV are to study the galaxy structure (Gonzalez et al. 2011, 2012), the hidden stellar clusters (Minniti et al. 2011; Borissova et al. 2011), and the nearby stars (Beamín et al. 2013; Ivanov et al. 2014), its exquisite depth and angular resolution make it an excellent tool to find and study extragalactic objects in the zone of avoidance. For example, Amores et al. (2012) identified 204 new galaxy candidates from the VVV photometry of 1.636 square degrees near the Galactic plane, which increases by more than an order of magnitude the surface density of known galaxies behind the Milky Way.

In this work, we report a NIR VVV identification of member galaxies in the new Suzaku J1759-3450 galaxy cluster. Colormagnitude and color-color diagrams of the sources in the cluster field were used to select candidate cluster members. We also identified the red-sequence formed by cluster ellipticals and 
Table 1. Observation parameters.

\begin{tabular}{lcccc}
\hline \hline Filter & $\begin{array}{c}\text { Exp.time } \\
\text { S }\end{array}$ & $\begin{array}{c}\text { NDIT } \\
\text { number }\end{array}$ & $\begin{array}{c}\text { Jitters } \\
\text { number }\end{array}$ & $\begin{array}{c}\text { Seeing } \\
\text { arcsec }\end{array}$ \\
\hline$J$ & 6 & 2 & 2 & 0.9 \\
$H$ & 4 & 1 & 2 & 0.8 \\
$K_{\mathrm{S}}$ & 4 & 1 & 2 & 0.8 \\
\hline
\end{tabular}

S0 galaxies. The presence of this red-sequence in the colormagnitude diagram is a clear sign of the existence of structure due to early-type cluster galaxy members occupying a welldefined region in the color space (Visvanathan \& Sandage 1977). Therefore, the red-sequence has been used as a galaxy-cluster detection method (Gladders \& Yee 2000), whose slope is related with the cluster redshift (Gladders et al. 1998; Lopez-Cruz et al. 2004; Stott et al. 2009).

The layout of the paper is as follows: The observational data are described in Sect. 2. In Sect. 3, the results of the analyzed data are shown. The discussion and conclusions are presented in Sects. 4 and 5, respectively. Throughout the paper, we use the following cosmological parameters: $H_{0}=67.3 \mathrm{~km} \mathrm{~s}^{-1} h^{-1}$, $\Omega_{\mathrm{M}}=0.315$, and $\Omega_{\lambda}=0.685$ (Planck Collaboration XVI 2014).

\section{Observational data}

\subsection{The VVV survey}

The VVV survey is a new Galactic NIR ESO public survey with the 4.1-m ESO VISTA (Visual and Infrared Survey Telescope for Astronomy; Emerson et al. 2004, 2006; Emerson \& Sutherland 2010) telescope, which is located at Cerro Paranal in Chile. It is equipped with a wide-field NIR camera VIRCAM (VISTA InfraRed CAMera; Dalton et al. 2006). The instrument has a field of view of $1.65^{\circ}$, and it is sensitive over a $\lambda=$ $0.9-2.4 \mu \mathrm{m}$ wavelength range. The VIRCAM has 16 Raytheon VIRGO $2048 \times 2048 \mathrm{HgCdTe}$ science detector, with a mean pixel scale of 0.34 arcsec $\mathrm{px}^{-1}$. Each individual detector covers $\sim 694 \times 694 \operatorname{arcsec}^{2}$ on the sky and offset exposures are taken to obtain contiguous coverage.

The VVV footprint covers a total of $~ 520$ square degrees on the sky, which are nearly equally split between the Milky Way bulge and the inner Southern disk. The survey has two components: (i) $Z Y J H K_{\mathrm{S}}$ bands; and (ii) up to 80 multi-epoch $K_{\mathrm{S}}$ observations spread over approximately $7-8$ years for variability and proper motion studies. More details can be found in Minniti et al. (2010) and Saito et al. (2012). The observations were processed within the VISTA Data Flow System (VDFS) pipeline at the Cambridge Astronomical Survey Unit (CASU; Lewis et al. 2010).

For our analysis, the $Z Y$ bands were omitted due to the larger extinction at these bands. The characteristics of the single epoch images in $J, H$, and $K_{\mathrm{S}}$ filters used in this work are summarized in Table 1. Finally, for the $K_{\mathrm{S}}$ band, we have built a deep image of the field that combines 30 different epochs. This last was possible since multi-epoch observations were obtained only for the $K_{\mathrm{S}}$ band for the goal of variability of the VVV survey.

\subsection{Source catalog}

It is well known that galactic morphology is correlated with the local density of galaxies (Dressler 1980). Therefore, in galaxy clusters, there is also a correlation between morphology and the projected distance from the cluster center. In this sense,
Whitmore \& Gilmore (1991) found that most of the variation occurs within $0.5 \mathrm{Mpc}$ of the cluster center, and that the effect is strongest in clusters with dominant galaxies with the percentage of ellipticals rising from $18 \%$ at $600 \mathrm{kpc}$ from the cluster center to $65 \%$ at distances of $100 \mathrm{kpc}$. Accordingly, Treu et al. (2003) found that the E+S0 component of galaxy clusters only within the central $200 \mathrm{kpc}$ become the dominant component.

Related with the identification of the red-sequence, to reduce the relative contamination of field galaxies, Gladders et al. (1998) used only the inner $0.5 \mathrm{~h}^{-1} \mathrm{Mpc}$ projected radius of the cluster centers to derives the slope of the red-sequence for a sample of 50 galaxy clusters, which spans a redshift range of $0<z<0.75$. Likewise, Stott et al. (2009) consider galaxies within $0.6 \mathrm{Mpc}$ radius of the cluster center by limiting the contamination from field galaxies for the study on the evolution of the red-sequence slope.

To minimize the contamination of field sources and to identify red-sequence elliptical galaxies, we concentrated on the very central region around the X-ray surface brightness peak of Suzaku J1759-3450 in this work, which falls on the VVV tile d261. We restricted our analysis to the inner $350 \mathrm{kpc}$ of the cluster center that corresponds to $\sim 1.5$ core radius, according to the $\beta$ model that is adopted for Mori et al. (2013) to fit the $\mathrm{X}$-ray profile of the surface brightness in the $0.5-10 \mathrm{keV}$ band.

Although CASU generates photometric catalogs for all VVV data, we preferred to create a new one by using SExtractor (Bertin \& Arnouts 1996), because it is better suited to treat extended sources. We run SExtractor in a double-image mode with the $K_{\mathrm{s}}$ deep image as a reference because of its better quality and lower susceptibility to extinction. An object was considered detected if the flux exceeded at least twice the local background noise level and spanned over at least ten connected pixels on the VVV images. The main entries in our final photometric catalog are

i) $J H K_{\mathrm{S}}$ magnitudes in three-pixel radius apertures. This is recommended to represent the flux for all the objects in the field of the VIRCAM and to calculate colors. The aperture correction was applied to these magnitudes by considering the apcor $^{1}$ parameter available in the header of the fits images. This correction, which is useful for point sources, also work well as a first order seeing correction for faint galaxies.

ii) $J H K_{\mathrm{S}}$ total magnitudes. For these, we used the MAG_AUTO from SExtractor, which is based by Kron's algorithm (Kron 1980).

iii) Star-galaxy separation parameters: CLASS_STAR and halflight radius parameter, $r_{1 / 2}$.

The 2MASS-based CASU-estimated zero points were used to flux calibrate the images. For the extinction correction, we used the high resolution extinction map of the Milky Way from Gonzalez et al. (2012). This reddening map has a resolution of 2 arcmin, and it is available via a web based tool BEAM (bulge extinction and metallicity) calculator ${ }^{2}$. This tool provides a lower limit for the mean extinction, $A_{K_{\mathrm{S}}}$ and the $E\left(J-K_{\mathrm{S}}\right)$ for a given set of coordinates and radius of the region. Finally, we adopted the Nishiyama et al. (2009) extinction law by obtaining the mean values of $A_{J}=0.26, A_{H}=0.14$ and $A_{K_{\mathrm{S}}}=0.09$. For this, analysis we used only objects with $K_{\mathrm{S}}=11-15.5 \mathrm{mag}$ to avoid stars that are saturated or too faint and blended objects.

\footnotetext{
1 http://casu.ast.cam.ac.uk/surveys-projects/vista/ technical/catalogue-generation

2 http://mill.astro.puc.cl/BEAM/calculator.php
} 


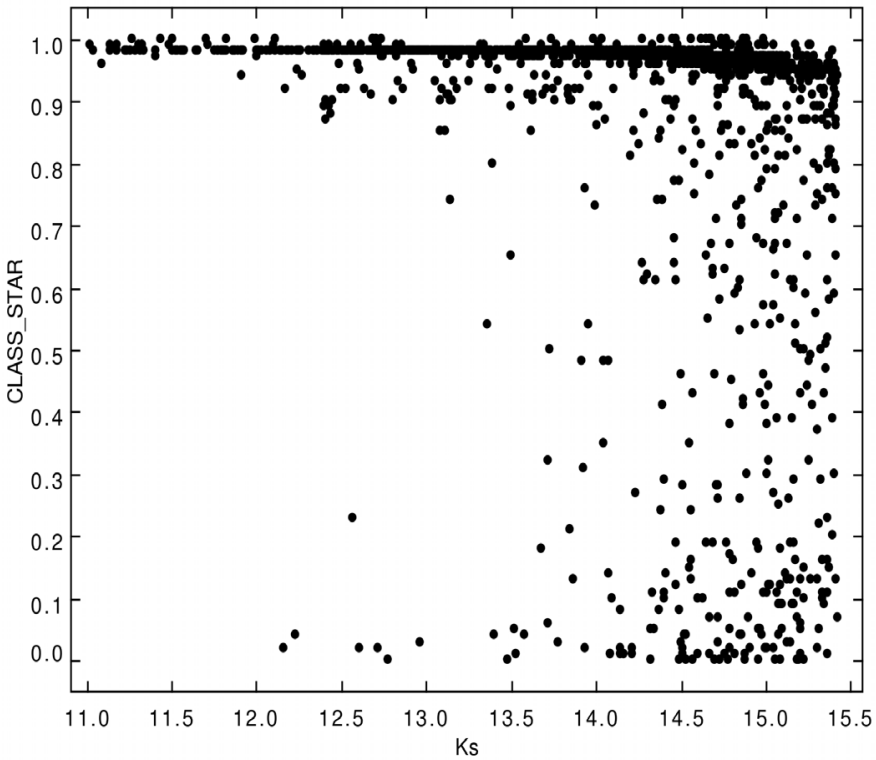

Fig. 1. The star-galaxy separation: SExtractor stellarity index CLASS_STAR versus the total $K_{\mathrm{S}}$ band source magnitude.

Our final catalog contains 1308 sources in the central region of the X-ray emission. The photometric calibration was crosschecked comparing the magnitudes of the point sources with those of the matching CASU sources. The average precision of our magnitudes respect to the CASU ones is $\sim 0.01 \mathrm{mag}$ in the three bands.

\section{Results}

\subsection{Star-galaxy separation}

Our star-galaxy classification was based on the SExtractor's stellarity index, CLASS_STAR, since the stars lie in a sequence near CLASS_STAR $=1$ and the galaxies near CLASS_STAR $=0$. The two sequences merge for fainter sources, and Bertin \& Arnouts (1996) showed that the stellarity index is strongly dependent on seeing in this regime. In Fig. 1, we show the relation between the CLASS_STAR parameter and the total $K_{\mathrm{S}}$ magnitude for our dataset. Taking the presence of the two sequences into account, we removed star-like sources from our catalog by adopting a conservative limit of CLASS_STAR $<0.5$.

The size versus magnitude diagram is another useful tool to perform star-galaxy separation. For this purpose, we used the half-light radius parameter, $r_{1 / 2}$. that measures the radius that encloses $50 \%$ of the object's total flux. For objects larger than the seeing, $r_{1 / 2}$ is independent of magnitude, placing the extended objects in a well-separated region of the diagram. The left panel of Fig. 2 shows the half-light radius versus total $K_{\mathrm{S}}$ magnitude for the whole sample of sources detected in the central region around the X-ray peak emission. The points are color-coded according to the stellarity parameter CLASS_STAR (the bar on the right) to underline the consistency of the two methods: the majority of the objects with CLASS_STAR $\sim 1$ approximately populate the stellar locus at $r_{1 / 2}<0.7$ arcsec (which corresponds to $\sim 2$ pixel radius), while sources with CLASS_STAR $<0.5$ tend to have larger $r_{1 / 2}$ as can be observed in the right panel of Fig. 2 .

Combining the two methods, we define as galaxy candidates those objects with a CLASS_STAR $<0.5$ and $r_{1 / 2}>0.7$ arcsec by obtaining a sample of 187 galaxy-like sources. Many of these objects could be unresolved double or multiple stars because of the extreme crowding in the Milky Way bulge. In the next section, colors are used to improve the galaxy candidate selection method mainly for those galaxies belonging to galaxy clusters.

\subsection{Red-sequence galaxies}

Galaxy clusters contain a well-defined, highly regular population of elliptical and lenticular galaxies that form a structure in their color-magnitude diagrams, known as a red-sequence (Gladders $\&$ Yee 2000). This alternative approach to identify clusters of galaxies has been proven to be highly successful by numerous authors (Gladders \& Yee 2000; Lopez-Cruz et al. 2004; Söchting et al. 2006), and we used it for a suitable identification of cluster members.

To further strengthen the identification of the galaxy cluster member candidates, we used a color selection criteria based on a red-sequence model derived from Bruzual \& Charlot (2003). The adopted model uses the Padova 94 (Fagotto et al. 1994a,b) evolutionary tracks and the Chabrier (2003) initial mass function with a passively evolving, instantaneous-burst stellar population with a redshift formation of $z=3$. The obtained mean red-sequence model is shown in Fig. 3, where the $J-K_{\mathrm{S}}$ versus $K_{\mathrm{S}}$ color-magnitude diagram of our field is shown. It was computed by averaging the models for different metallicities at the redshift of the galaxy cluster, $z=0.13$.

Based on the aforementioned, we defined as cluster member those galaxies within $\pm 5 \sigma$ (Fig. 3) from the adopted redsequence model in the color-magnitude diagram. It is important to emphasize that color gradients could vary from one galaxy to another, so to obtain unbiased colors of the galaxy candidates we used fixed aperture magnitudes for all filters. This color cut yielded 22 final galaxy candidates.

The density map in the color-magnitude diagram of Fig. 3 represents the whole sample of objects in the central region of the X-ray peak emission. On the other hand, the points in the figure indicate those source that satisfy the CLASS_STAR $<0.5$ and $r_{1 / 2}>0.7 \operatorname{arcsec}$ criteria. From this figure, it is possible to observe two well-defined regions in the color-magnitude diagram: one horizontal sequence at $J-K_{\mathrm{S}}<1.0$ (shown by the color density map) that corresponds to the stellar sequence of galactic stars, probably with types later than G5 and earlier than K5 (Finlator et al. 2000), and the other that corresponds to the objects defined as galaxy candidates, which are located mainly in the region close to the modeled red-sequence. The candidate galaxies in the region of the red-sequence could have an appearance corresponding to early type objects, such as elliptical and lenticular galaxies.

Next, we visually inspected these galaxy candidates on a false-color multiband image (built from $J, H, K$ s frames) using Aladin (Bonnarel et al. 2000), to identify extended sources with the typical galactic features: color, sizes and ellipticity. We verified the galactic features of 15 candidates, whereas seven remained unclassified, typically because they were too faint and an objective classification is not possible. Moreover, from these 15 objects, we classified them in two categories: Type I sources as those with clear galaxy features that result in 12 candidate galaxies in this category; and Type II as the remaining sources with features that are not completely clear expected for galaxies. The visually confirmed galaxies belonging to the redsequence can be seen in Fig. 3 .

The cluster member galaxy candidates are, indeed, red in all colors, as can be clearly seen from the color-color diagram 


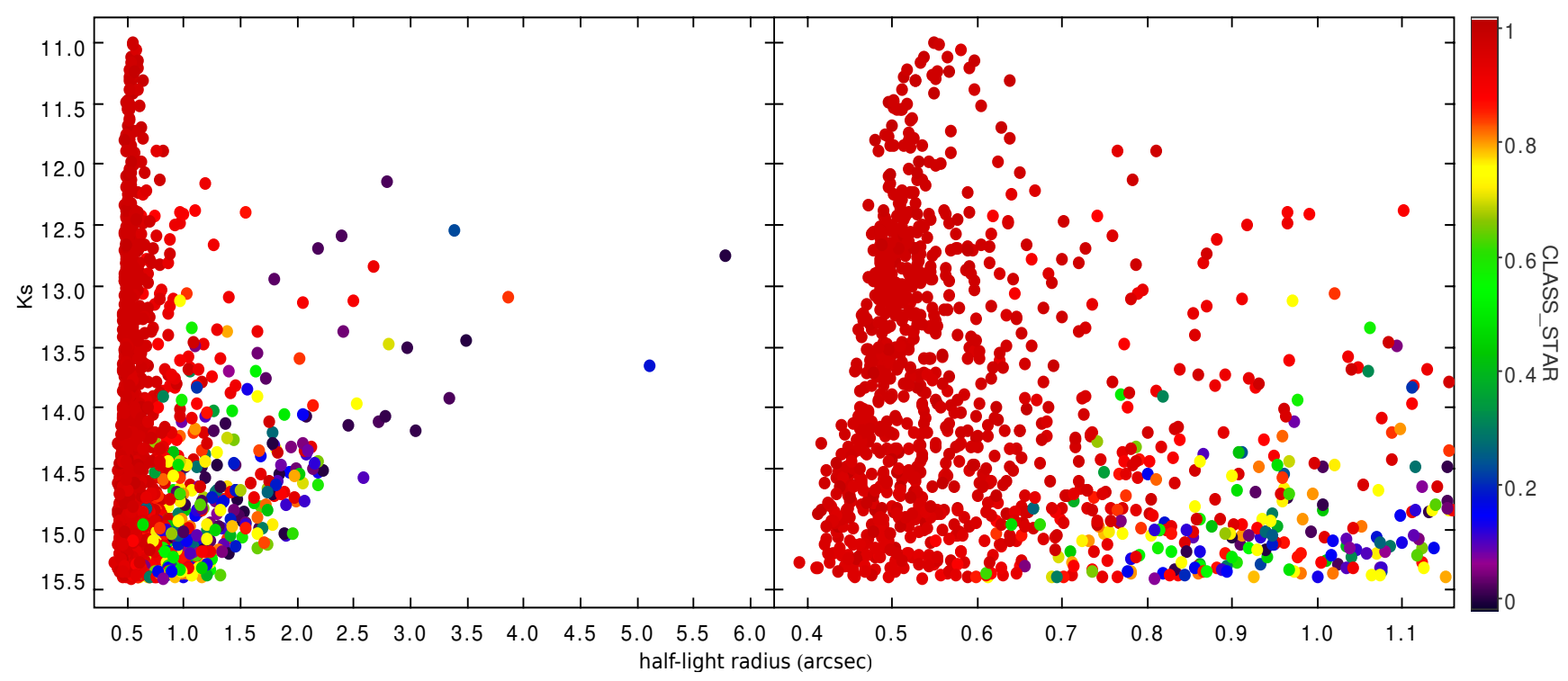

Fig. 2. Left: half-light size $r_{1 / 2}$ versus $K_{\mathrm{S}}$ magnitude diagram for the whole sample of detected sources in the central region. Right: enlargement of the left panel for low values of $r_{1 / 2}$ radius. The points are color-coded, according to the SExtractor's stellarity parameter CLASS_STAR (the bar on the right).

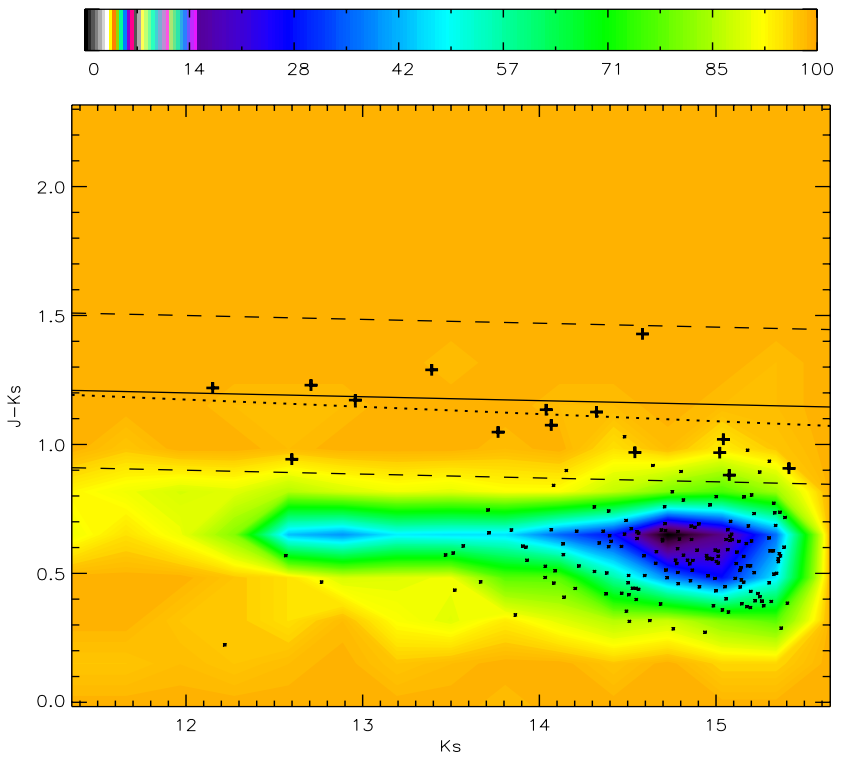

Fig. 3. Color-magnitude diagram $J-K_{\mathrm{S}}$ versus $K_{\mathrm{S}}$. The density map shows the whole sample in the central region of the cluster (color scale corresponds to the percentage of objects with respect to the total number, as shown in the key). The red-sequence model is represented by a solid line and the $\pm 5 \sigma$ around the model by dashed lines. The dots correspond to the sources that met the selection criteria of Sect. 3.1. The crosses mark the red-sequence galaxies with visual confirmation. The red sequence, fitted to the bright galaxy cluster member candidates, is shown by the dotted line.

shown in Fig. 4; they occupy a different locus than the foreground stars. This is consistent with the results of Amores et al. (2012), who visually identified galaxies behind the Galactic plane in the VVV survey, and used color-color diagrams to confirm the galaxy-star separation.

Finally, Fig. 5 shows a false-color multi-band $(J, H, K s)$ image, as centered in the X-ray peak emission, where the five brightest galaxies can be found very close to this peak. From this image, it is possible to observe that the galaxy cluster member candidates show redder colors with respect to the foreground

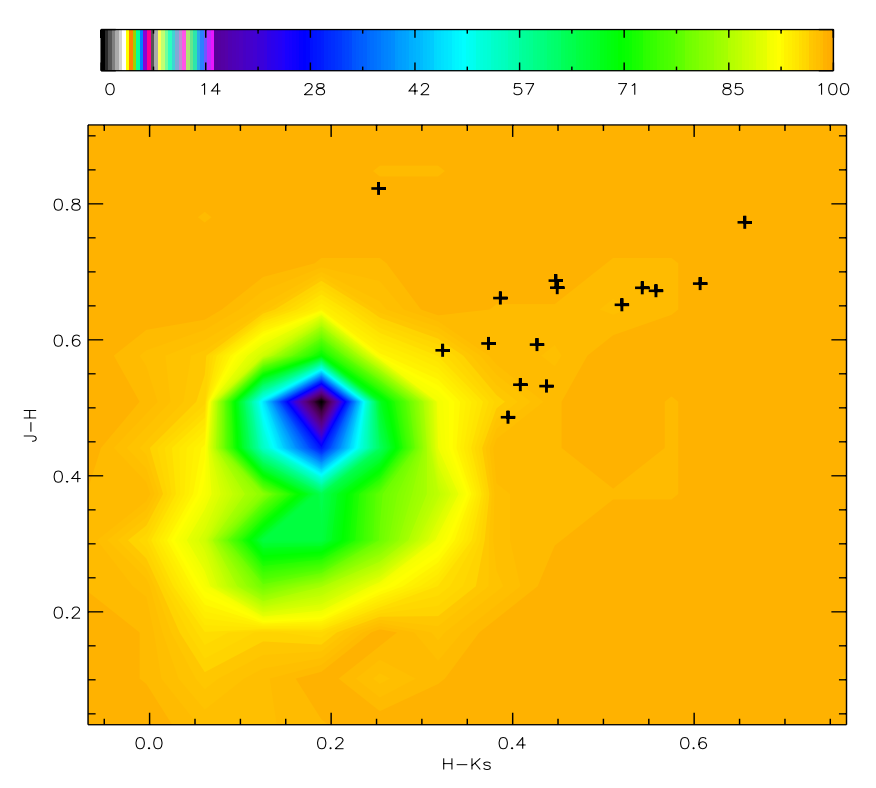

Fig. 4. Color-color diagram $J-H$ versus $H-K_{\mathrm{S}}$. The density map shows the whole sample in the central region of the cluster (color scale corresponds to the percentage of objects with respect to the total number, as shown in the key). The crosses mark the red-sequence galaxies, as selected from Fig. 3 .

stars and also extended morphology characteristic of galaxies. Futhermore, the area corresponding to the core radius, according to the $\beta$ model adopted for Mori et al. (2013) to fit the X-ray profile of the surface brightness, is shown.

Table 2 lists the cluster galaxy candidate parameters that are ordered by $K$ s total magnitude. The list includes an identification number, coordinates, aperture magnitudes, total magnitudes, and classification.

Although we cannot estimate the real contamination of background galaxies without redshift measurements, the restriction of the analysis to the central region of the galaxy cluster favors elliptical and S0 galaxies with typical red colors minimizes the background galaxy contamination. For instance, the redsequence has been used as an efficient method to detect galaxy 
Georgina Coldwell et al.: Cluster of galaxies behind the Galactic bulge
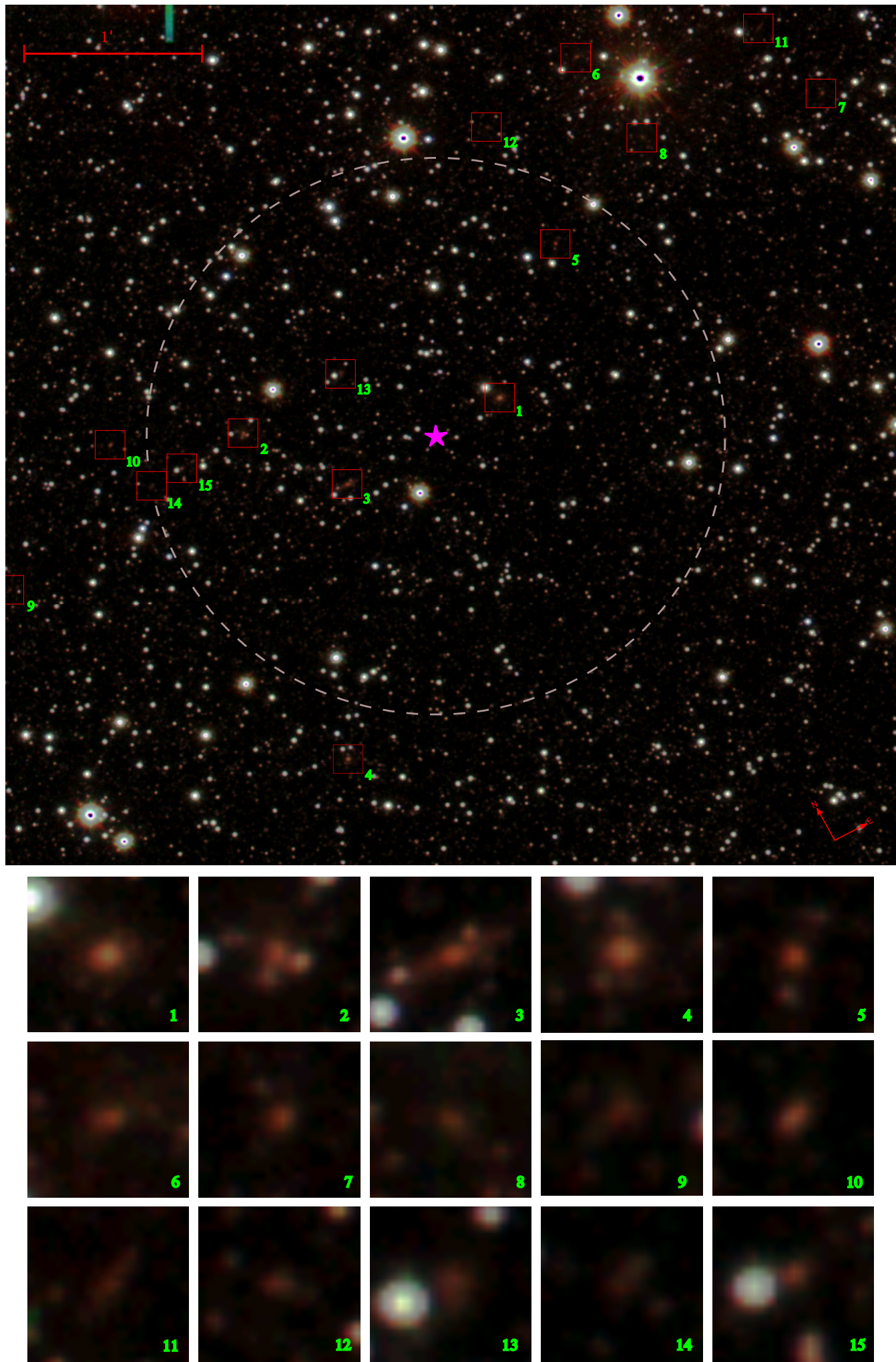

Fig. 5. False-color $J$ (blue), $H$ (green), and $K_{\mathrm{S}}$ (red) image of the cluster region. The star symbol indicates the peak of the X-ray emission. All the candidate galaxies are enumerated in the images. The grey circular dashed line shows the area corresponding to the core radius of the X-ray emission, $r_{\mathrm{c}}=1.61 \mathrm{arcmin}$. A scale bar is shown at the top left. The postage stamp images of every galaxy candidate with the identification number are at the bottom.

clusters at optical and infrared wavelengths, with a contamination of less than 5\% (Gladders \& Yee 2000). More recently, Barkhouse et al. (2006) uses the red-sequence to improve the Voronoi tessellation and percolation technique by significantly reducing the contamination.

\section{Discussion}

The analysis reported in the previous sections demonstrated that the field of Suzaku J1759-3450 contains multiple galaxy candidates that show the colors and magnitudes cluster members, 
Table 2. Cluster galaxy candidates.

\begin{tabular}{|c|c|c|c|c|c|c|c|c|c|}
\hline ID & $\begin{array}{l}\text { RA(J2000) } \\
\text { hh:mm:ss.ss }\end{array}$ & $\begin{array}{l}\operatorname{Dec}(J 2000) \\
\text { dd:mm:ss.ss }\end{array}$ & $\begin{array}{l}J(3) \\
\text { mag }\end{array}$ & $\begin{array}{l}H(3) \\
\text { mag }\end{array}$ & $\begin{array}{c}K_{\mathrm{S}}(3) \\
\mathrm{mag}\end{array}$ & $\begin{array}{c}J \\
\mathrm{mag}\end{array}$ & $\begin{array}{c}H \\
\mathrm{mag}\end{array}$ & $\begin{array}{c}K_{\mathrm{S}} \\
\mathrm{mag}\end{array}$ & Class \\
\hline 1 & 17:59:19.44 & $-34: 50: 18.7$ & $15.19 \pm 0.03$ & $14.52 \pm 0.02$ & $13.98 \pm 0.02$ & $13.00 \pm 0.02$ & $12.50 \pm 0.02$ & $12.12 \pm 0.02$ & I \\
\hline 2 & $17: 59: 12.98$ & $-34: 49: 48.5$ & $15.30 \pm 0.03$ & $14.76 \pm 0.02$ & $14.36 \pm 0.02$ & $13.39 \pm 0.02$ & $12.95 \pm 0.02$ & $12.62 \pm 0.02$ & I \\
\hline 3 & $17: 59: 14.75$ & $-34: 50: 19.9$ & $15.64 \pm 0.04$ & $14.97 \pm 0.03$ & $14.41 \pm 0.03$ & $13.40 \pm 0.02$ & $13.06 \pm 0.02$ & $12.71 \pm 0.02$ & I \\
\hline 4 & 17:59:10.97 & $-34: 51: 42.2$ & $15.50 \pm 0.03$ & $14.85 \pm 0.02$ & $14.33 \pm 0.02$ & $13.70 \pm 0.02$ & $13.27 \pm 0.02$ & $12.96 \pm 0.02$ & I \\
\hline 5 & $17: 59: 22.85$ & $-34: 49: 42.0$ & $16.21 \pm 0.06$ & $15.53 \pm 0.04$ & $14.92 \pm 0.04$ & $14.12 \pm 0.03$ & $13.77 \pm 0.03$ & $13.39 \pm 0.03$ & I \\
\hline 6 & $17: 59: 25.91$ & $-34: 48: 49.5$ & $16.21 \pm 0.05$ & $15.55 \pm 0.04$ & $15.16 \pm 0.04$ & $14.91 \pm 0.06$ & $14.30 \pm 0.05$ & $13.77 \pm 0.04$ & I \\
\hline 7 & $17: 59: 31.11$ & $-34: 49: 39.5$ & $16.28 \pm 0.06$ & $15.60 \pm 0.04$ & $15.15 \pm 0.04$ & $14.58 \pm 0.04$ & $14.22 \pm 0.04$ & $14.04 \pm 0.05$ & I \\
\hline 8 & $17: 59: 26.33$ & $-34: 49: 24.0$ & $16.53 \pm 0.07$ & $15.70 \pm 0.05$ & $15.45 \pm 0.05$ & $14.69 \pm 0.04$ & $13.99 \pm 0.03$ & $14.07 \pm 0.05$ & I \\
\hline 9 & 17:59:05.36 & $-34: 49: 56.9$ & $16.73 \pm 0.08$ & $16.05 \pm 0.06$ & $15.60 \pm 0.06$ & $15.24 \pm 0.06$ & $14.63 \pm 0.05$ & $14.32 \pm 0.05$ & II \\
\hline 10 & 17:59:09.71 & $-34: 49: 30.0$ & $15.94 \pm 0.05$ & $15.40 \pm 0.04$ & $14.97 \pm 0.04$ & $15.35 \pm 0.05$ & $14.82 \pm 0.05$ & $14.54 \pm 0.05$ & I \\
\hline 11 & $17: 59: 30.54$ & $-34: 49: 10.0$ & $17.01 \pm 0.10$ & $16.24 \pm 0.07$ & $15.58 \pm 0.06$ & $15.58 \pm 0.07$ & $15.14 \pm 0.07$ & $14.58 \pm 0.06$ & I \\
\hline 12 & $17: 59: 22.85$ & $-34: 48: 56.2$ & $16.47 \pm 0.06$ & $15.88 \pm 0.05$ & $15.51 \pm 0.05$ & $15.62 \pm 0.06$ & $15.22 \pm 0.06$ & $15.02 \pm 0.07$ & II \\
\hline 13 & $17: 59: 16.07$ & $-34: 49: 45.6$ & $16.18 \pm 0.05$ & $15.59 \pm 0.04$ & $15.16 \pm 0.04$ & $16.05 \pm 0.06$ & $15.45 \pm 0.05$ & $15.04 \pm 0.05$ & I \\
\hline 14 & 17:59:10.11 & $-34: 49: 49.2$ & $16.53 \pm 0.07$ & $16.05 \pm 0.06$ & $15.65 \pm 0.06$ & $15.53 \pm 0.07$ & $15.28 \pm 0.08$ & $15.07 \pm 0.09$ & II \\
\hline 15 & 17:59:11.06 & $-34: 49: 48.5$ & $16.55 \pm 0.07$ & $15.97 \pm 0.06$ & $15.65 \pm 0.06$ & $16.42 \pm 0.07$ & $16.01 \pm 0.06$ & $15.41 \pm 0.06$ & I \\
\hline
\end{tabular}

Notes. $\mathrm{J}(3), \mathrm{H}(3)$, and $K_{\mathrm{S}}(3)$ are magnitudes in apertures with 3 pixels of radius, and $\mathrm{JHK}_{\mathrm{S}}$ correspond to total magnitudes. The class parameter indicates clear (I) or unclear (II) galaxy features.

including the brightest galaxy, with $K_{\mathrm{S}} \sim 12.1 \mathrm{mag}$, and four other prominent objects in the magnitude range $12.5<K_{\mathrm{S}}<$ 13.5. Brough et al. (2002) indicates that the brightest cluster galaxies (BCGs) in clusters with $\mathrm{X}$-ray luminosity $L_{\mathrm{x}}(0.3-$ $3.5 \mathrm{keV})>1.9 \times 10^{44} \mathrm{erg} \mathrm{s}^{-1}$ have apparent magnitudes around $K_{\mathrm{S}} \sim 12 \mathrm{mag}$, consistent with our measurements. Moreover, the apparent magnitude of the BCG is consistent with an absolute magnitude of $M_{K_{\mathrm{S}}} \sim-25.6$, if we assume that this galaxy is a member of a galaxy cluster at redshift $z=0.13$, also in agreement with Brough et al. (2002). These results reinforce the assumption that the red-sequence detected indeed corresponds to the new galaxy cluster, reported by Mori et al. (2013).

Considering the visual appearance of the BCG corresponding to a very luminous elliptical galaxy, half magnitude brighter than the second brightest galaxy, the large diffuse halo, and the proximity to the X-ray emission peak $(\sim 25 \mathrm{arcsec})$ we could speculate that this is a $\mathrm{cD}$ galaxy.

In addition to the modeled red-sequence, we also calculated the red-sequence slope by using only the visually confirmed galaxies, which are brighter than $K_{\mathrm{S}}=14.1$. These correspond to galaxies that are two magnitudes fainter than the brightest one to minimize the contamination that is more likely for the faintest galaxies. This empiric red-sequence is shown in Fig. 3 by showing a good agreement with the modeled one. It is important to highlight that a random selection of galaxies at different redshifts does not form a coherent red sequence (Gladders \& Yee 2000); therefore, the projection effect would not significantly affect this selection criteria. Anyway, spectroscopic measurement of the redshifts of the newly identified cluster members are extremely useful to confirm these results. The obtained value of the slope, $k_{J K_{\mathrm{S}}}=-0.028 \pm 0.011$ is consistent with that found by Stott et al. (2009) for three galaxy clusters at $z \sim 0.13$.

It is worth mentioning that the detected candidates of a galaxy cluster member have an asymmetric spatial distribution with respect to the position of the X-ray peak emission. Instead, the galaxies are preferentially located on one side of the X-ray peak emission. In this sense, Forman \& Jones (1982) and Jones \& Forman (1984) propose a two-dimensional classification scheme for the X-ray morphology of the intracluster gas that represents a sequence of cluster evolution. Moreover, the presence or absence of a central, dominant galaxy in the cluster is considered in the classification scheme of Forman \& Jones (1982). The authors classify the clusters as either being early if the overall X-ray surface brightness distribution is irregular or evolved if it is regular. In addition, clusters containing a central dominant galaxies are classified as X-ray dominant (XD), while those without such a galaxy are classified as non-X-ray dominant (nXD). The $\mathrm{nXD}$ clusters have larger X-ray core radii, and there is no strong $\mathrm{X}$-ray emission associated with any individual galaxy in these systems. That is, the galaxy cluster could be $\mathrm{X}$-ray dominant or non-X-ray dominant for the regular or irregular X-ray surface brightness.

As it was mentioned, the cluster in this particular case has a regular X-ray morphology without a central dominant galaxy in the X-ray peak emission. Thus, Suzaku J1759-3450 can be classified as a regular nXD cluster. Several galaxy clusters have a similar characteristic, including A1656 (Abramopoulos et al. 1981), A576 (White \& Silk 1980), A2255, A2256 (Forman \& Jones 1982), and CA0340-538 (Ku et al. 1983). This feature indicates that the galaxy cluster is still evolving and suffering dynamical processes. In the same sense, even with a approximately circular X-ray morphology, Mori et al. (2013) found that the peak position of the surface brightness is slightly shifted from the center of the circular emission in the southeast direction, suggesting that the cluster dynamical relaxation has not taken place yet.

\section{Conclusions}

Summarizing the above, we used the VVV survey data to obtain deep NIR photometry of objects in the vicinity of the recently identified galaxy cluster, Suzaku J1759-3450, at $z=0.13$. At least fifteen galaxies were detected within a projected distance of $350 \mathrm{kpc}$ from the central peak of the X-ray peak emission, and five of them are extremely bright.

All these cluster member candidates have the typical colors and magnitudes of galaxies at redshift $z=0.13$. The candidate BCG is more than a half magnitude brighter than the next brightest galaxy candidate, indicating that it may be a cD galaxy. These results lead us to conclude that Suzaku J1759-3450 is indeed an obscured galaxy cluster at $z=0.13$. Spectroscopic observations 
are needed to measure the redshifts of the newly identified cluster members and to study their stellar populations in detail.

Acknowledgements. We would like to thanks to anonymous referee for the comments that helped to improve the paper. G.C., S.A. and F.D. acknowledge the support from CONICET through grant PIP 2012-2014, GI 11220110100298. Support for M.H. has been provided by the BASAL Center for Astrophysics and Associated Technologies PFB-06, the FONDAP Center for Astrophysics No. 15010003, and the Ministry for the Economy, Development and Tourisms Programa Iniciativa Científica Milenio through grant P07-021-F, awarded to The Milky Way Millenium Nucleus. D.M. is supported by Project IC120009 Millennium Institute of Astrophysics (MAS) of Iniciativa Científica Milenio del Ministerio de Economía, Fomento y Turismo, by the Basal Center for Astrophysics and Associated Technologies (CATA) PFB-06, and by Fondecyt Project No. 1130196. G.V. acknowledge the support from Agencia Nacional de Promoción Científica y Tecnológica, PICT 2010 Bicentenario N0680.

\section{References}

Abramopoulos, F., Chanan, G. A., \& Ku, W. H. M. 1981, ApJ, 248, 429

Amores, E., Sodre, L., Minniti, D., et al. 2012, AJ, 144, 127

Bahcall, N. A., Dong, F., Bode, P., et al. 2003, ApJ, 585, 182

Barkhouse, W. A., Green, P. J., Vikhlinin, A., et al. 2006, ApJ, 645, 955

Beamín, J. C., Minniti, D., Gromadzki, M., et al. 2013, A\&A, 557, L8

Bertin, E., \& Arnouts, S. 1996, A\&AS, 117, 393

Bonnarel, F., Fernique, P., Bienaymé, O., et al. 2000, A\&AS, 143, 33

Borissova, J., Bonatto, C., Kurtev, R., et al. 2011, A\&A, 532, A131

Brough, S., Collins, C. A., Burke, D. J., Mann, R. G., \& Lynam, P. D. 2002, MNRAS, 329, 53

Bruzual, G., \& Charlot, S. 2003, MNRAS, 344, 1000

Chabrier, G. 2003, PASP, 115, 763

Dalton, G. B., Caldwell, M., Ward, A. K., et al. 2006, Proc. SPIE, 6269, 30

Dressler, A. 1980, ApJ, 236, 351

Emerson, J. P., \& Sutherland, W. J. 2010, Proc. SPIE, 7733

Emerson, J. P., Sutherland, W. J., McPherson, A. M., et al. 2004, The Messenger, 117,27

Emerson, J. P., McPherson, A. M., Sutherland, W. J., et al. 2006, The Messenger, 126,41

Fagotto, F., Bressan, A., Bertelli, G., \& Chiosi, C. 1994a, A\&AS, 104, 365
Fagotto, F., Bressan, A., Bertelli, G., \& Chiosi, C. 1994b, A\&AS, 105, 29 Finlator, K., Ivezić, Ž., Fan, X., et al. 2000, AJ, 120, 2615

Finoguenov, A., Guzzo, L., Hasinger, G., et al. 2007, ApJS, 172, 182

Forman, W., \& Jones, C. 1982, ARA\&A, 20, 547

Gladders, M. D., \& Yee, H. K. C. 2000, AJ, 120, 2148

Gladders, M. D., Lopez-Cruz, O., Yee, H. C. K., \& Kodama, T. 1998, ApJ, 501, 571

Gladders, M. D., Yee, H. K. C., Majumdar, S., et al. 2007, ApJ, 655, 128

Gonzalez, O. A., Rejkuba, M., Zoccali, M., Valenti, E., \& Minniti, D. 2011 A\&A 534, A3

Gonzalez, O. A., Rejkuba, M., Zoccali, M., et al. 2012, A\&A, 543, A13

Gursky, H., Levinson, R., Kellogg, E., et al. 1972, ApJ, 173, 99

Ivanov, V. D., Minniti, D., Hempel, M., et al. 2014, A\&A, 560, A21

Jones, C., \& Forman, W. 1984, ApJ, 276, 38

Kraan-Korteweg, R. C., \& Lahav O. 2000, A\&ARv, 10, 211

Kron, R. G. 1980, ApJ, 43, 305

Ku, W. H. M., Abramopoulos, F., Nulsen, P. E. J., et al. 1983, MNRAS, 203, 253

Lewis, J. R., Irwin, M., \& Bunclark, P. 2010, ASP Conf., 434, 91

Lopez-Cruz, O., Barkhouse, W. A., \& Yee, H. K. C. 2004, ApJ, 644, 759

Minniti, D., Lucas, P. W., Emerson, P. J., et al. 2010, New Astron., 15, 433

Minniti, D., Hemoel, M., Toledo, I., et al. 2011, A\&A, 527, A81

Mori, H., Maeda, Y., Furuzawa, A., Haba, Y., \& Ueda, Y. 2013, PASJ, 65, 102

Nishiyama, S., Tamura, M., Hatano, H., et al. 2009, ApJ, 696, 1407

Pierre, M., Pacaud, F., Duc, P. A., et al., 2006, MNRAS, 372, 591

Planck Collaboration XVI. 2014, A\&A, in press, DOI: 10.1051/0004-6361/201321591

Romer, A. K., Viana, P. T. P., Liddle, A. R., \& Mann, R. G. 2001, ApJ, 547, 594

Rozo, E., Wechsler, R. H., Rykoff, E. S., et al. 2010, ApJ, 708, 645

Saito, R. K., Hempel, M., Minniti, D., et al. 2012, A\&A, 537, A107

Söchting, I. K., Huber, M. E., Clowes, R. G., \& Howell, S. B. 2006, MNRAS, 369,1334

Stott, J. P., Pimbblet, K. A., Edge, A. C., Smith, G. P., \& Wardlow, J. L. 2009, MNRAS, 394, 2098

Treu, T., Ellis, R. S., Kneib, J. P., et al. 2003, ApJ, 591, 53

Visvanathan, N., \& Sandage, A. 1977, ApJ, 216, 214

Voges, W., Aschenbach, B., Boller, Th., et al. 1999, A\&A, 349, 389

White, S. D. M., \& Silk, J. 1980, ApJ, 241, 864

Whitmore, B. C., \& Gilmore, D. M. 1991, ApJ, 367, 64

Woudt, P. A., Kraan-Korteweg, R. C., Cayatte, V., Balkowski, C., \& Felenbok, P. 2004, A\&A, 415, 9 